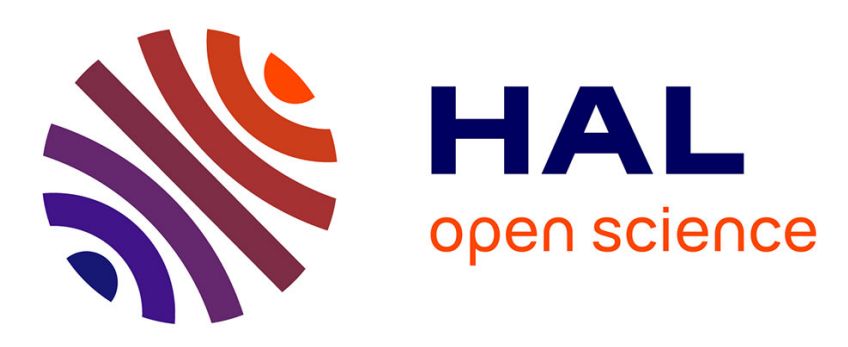

\title{
Movie Script Similarity Using Multilayer Network Portrait Divergence
}

\author{
Majda Lafhel, Hocine Cherifi, Benjamin Renoust, Mohammed El hassouni, \\ Youssef Mourchid
}

\section{- To cite this version:}

Majda Lafhel, Hocine Cherifi, Benjamin Renoust, Mohammed El hassouni, Youssef Mourchid. Movie Script Similarity Using Multilayer Network Portrait Divergence. Complex Networks \& Their Applications IX pp 284-295, pp.284 - 295, 2020, 10.1007/978-3-030-65347-7_24 . hal-03201616

\section{HAL Id: hal-03201616 https://hal.science/hal-03201616}

Submitted on 19 Apr 2021

HAL is a multi-disciplinary open access archive for the deposit and dissemination of scientific research documents, whether they are published or not. The documents may come from teaching and research institutions in France or abroad, or from public or private research centers.
L'archive ouverte pluridisciplinaire HAL, est destinée au dépôt et à la diffusion de documents scientifiques de niveau recherche, publiés ou non, émanant des établissements d'enseignement et de recherche français ou étrangers, des laboratoires publics ou privés. 


\section{Movie Script Similarity Using Multilayer Network Portrait Divergence}

Chapter · January 2021

DOI: 10.1007/978-3-030-65347-7_24

CITATIONS

5 authors, including:

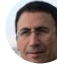

Hocine Cherifi

University of Burgundy

203 PUBLICATIONS 1,465 CITATIONS

SEE PROFILE

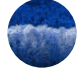

Mohammed El Hassouni

Mohammed V University in Rabat

171 PUBLICATIONS 761 CITATIONS

SEE PROFILE

Some of the authors of this publication are also working on these related projects:

Project Shape analysis View project

Project Image Segmentation View project
READS

38

Benjamin Renoust

National Institute of Informatics

49 PUBLICATIONS 176 CITATIONS

SEE PROFILE

Youssef Mourchid

Yncréa Ouest/ISEN Brest-France

18 PUBLICATIONS 46 CITATIONS

SEE PROFILE 


\title{
Movie Script Similarity using Multilayer Network Portrait Divergence
}

\author{
Majda Lafhel ${ }^{1}$, Hocine Cherifi ${ }^{2}$, Benjamin Renoust ${ }^{3}$, Mohammed El Hassouni ${ }^{1}$, \\ and Youssef Mourchid ${ }^{4}$ \\ 1 LRIT URAC 29, Mohammed V University \\ majdalafhel1@gmail.com, mohamed.elhassouni@gmail.com , \\ 2 LIB, University of Burgundy \\ hocine.cherifi@u-bourgogne.fr \\ 3 Institute for Datability Science, Osaka University \\ renoust@ids.osaka-u.ac.jp \\ 4 L@bisen, Yncrea Ouest, Brest, France \\ youssefmour@gmail.com
}

\begin{abstract}
This paper addresses the question of movie similarity through multilayer graph similarity measures. Recent work has shown how to construct multilayer networks using movie scripts, and how they capture different aspects of the stories. Based on this modeling, we propose to rely on the multilayer structure and compute different similarities, so we may compare movies, not from their visual content, summary, or actors, but actually from their own storyboard. We propose to do so using "portrait divergence", which has been recently introduced to compute graph distances from summarizing graph characteristics. We illustrate our approach on the series of six Star Wars movies.
\end{abstract}

Keywords: Multilayer networks, Movies, Network portrait, Network similarity.

\section{Introduction}

Network models have been increasingly used to support the analysis of stories [1], such as novels and plays [23] 26] [14, famous TV series [24, news [20, 21, and movies [18] 15] 16. However, most of these models only focus on one facet of the movie story. Indeed, usually, they capture interactions between the characters at play [11 to bring out a global picture of the story content. Other works went beyond by introducing other semantic elements such as scenes and dialogues [24]. Nevertheless, they have always captured the information in a single layer network or a bipartite graph. To enrich the representation, we have previously proposed a multilayer network model that captures key elements of the movie story [15] [16]. It encompasses the single network analysis based either on characters or scenes and proposes new topological analysis tools.

Recently, various studies are being conducted to measure the similarity of movie stories [13] 12] focusing on characters interactions. This work, aims at 
quantifying similarity between movies by incorporating knowledge extracted from complementary aspects of a story, including character, dialogues, and locations. To do so, rather than relying on a single-layer network, a multilayer network extracted from the movie script is exploited. It combines semantically extracted characters, keywords, and locations, allowing to compute similarity between the corresponding networks. Starting from the previously proposed model [15] 16] the multilayer network is automatically extracted from the scripts of the movies to compare. At this point the similarity issue reduces to compute the similarities between the layers. Plenty of methods have been proposed in the literature for comparing networks 25 . Here, a recent method introduced by Bagrow et al. [2] is used. The so-called Network Portrait Divergence is based on the network portrait [3], which characterizes a network by determining its degree distribution at various distances. Experiments performed on the Star Wars series show encouraging results that match up with the perceptual analysis.

The rest of the paper is organized as follows. Section 2, summarizes the multilayer movie script model and its extraction process, together with network portrait and its divergence. Section 3 starts with introducing the data then results of the evaluation are discussed. Conclusion and discussions are reported in Section 4 .

\section{Background}

\subsection{Extracting multilayer networks from movie scripts}

The multilayer network model [15] considers three types of entities, each forming a unique layer: characters $C$, keywords $K$, and locations $L$, together with their interactions. Intralayer interactions: $C-C$ when two characters share a conversation; $K-K$ when two keywords co-occur in a conversation; $L-L$ when two locations are in successive scenes. Interlayer interactions also exist: $C-K$ when a character pronounces a keyword; $C-L$ when a character is in a location; $K-L$ when a keyword is pronounced in a location.

A movie script is a semi-structured text containing all technical information concerning scenes, dialogues, and settings. It is divided into scenes delimited by scene heading that specify the physical spaces (INT or EXT), location, and the time (DAY or NIGHT).

The first step of processing consists in dividing the script into scenes based on the scenes headings. Fig. 1 illustrates a scene. Locations are extracted from the scene heading. Characters are then extracted as dialogues header (in capital). Finally, keywords are extracted from dialogues by applying Latent Dirichlet Allocation (LDA) 4]. Named Entity Recognition (NER) [17] is further applied to extract character and location from the keywords.

From Fig. 1, the following links and entities can be extracted: $C-C: A N A K I N$ and SHMI; K-K: Mom and Annie; L-L: TUSKEN RAIDER HUTT and GEONOSIS, then GEONOSIS and TATOOINE; C-K:ANAKIN and Mom; $C-L: A N A K I N$ and TUSKEN RAIDER HUTT, then SHMI and TUSKEN RAIDER HUTT; K - L:Mom and TUSKEN RAIDER HUTT. 


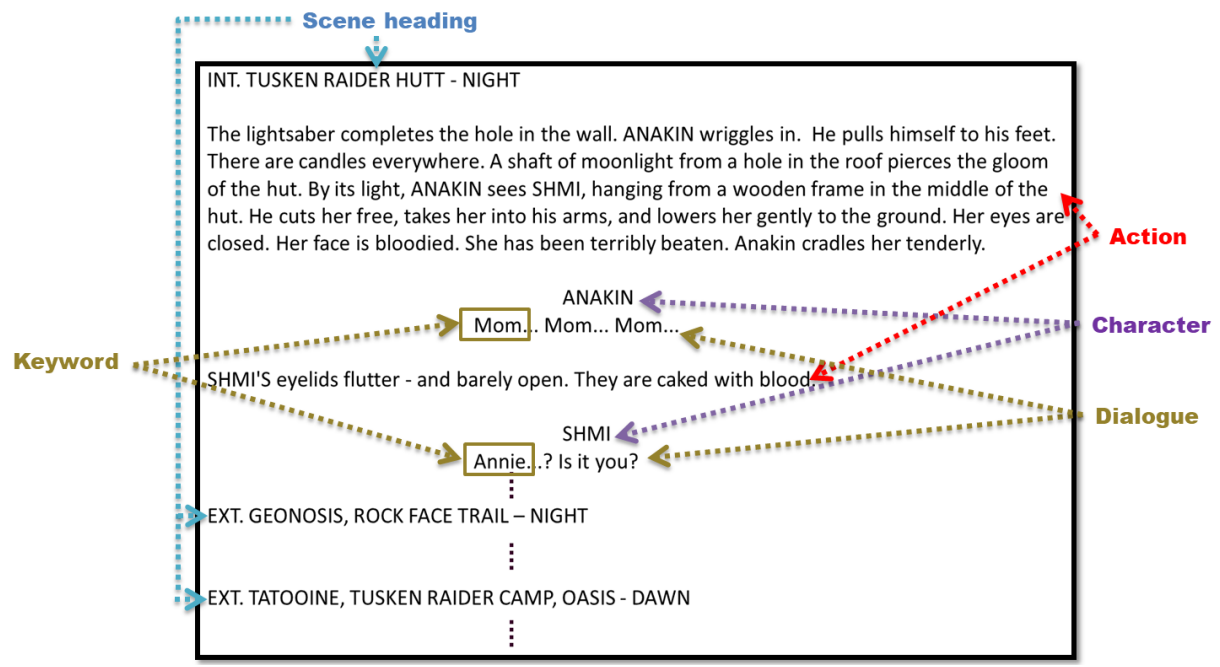

Fig. 1: Excerpt of Attack of the Clones script describing one scene.

\subsection{Network comparison using portrait and portrait divergence}

In 2008, Bagrow introduced Network Portraits 3 to summarize large complex networks. The Network Portrait is a matrix B with $B_{l, k}$ elements such as defined in eq. (1). Given a network $G$ with $N$ nodes, $k$ is a number of nodes such that $0 \leq k \leq N$, and $l$ is the shortest path length between two nodes, such that $0 \leq l \leq d$ (graph diameter).

More formally:

$$
B_{l, k} \equiv \text { the number of nodes connected with k nodes at distance } 1
$$

Each row in $B$ represents the probability distribution $P(k \mid l)$ such that $k$ nodes are reachable at distance $l$ from a randomly chosen node:

$$
P(k \mid l)=\frac{1}{N} B_{l, k} \quad B_{1, k}=N \mathbf{P}(\mathbf{k})
$$

$B$ is a signature of $G$. Bagrow then introduced a distance measure between two networks $G$ and $G^{\prime}$, the Network Portrait Divergence 2] based on this signature.

Let $B$ and $B^{\prime}$ be the portraits associated with $G$ and $G^{\prime}$ respectively. The portrait divergence computes first the probability distribution of nodes based on the matrices $B$ and $B^{\prime}$, such as in $e q$. 3. 4. Then, the distance between the probability distributions is computed using the Jensen Shannon divergence $D_{J S}$, such as in $e q .5$.

Let $G$ be a network of node size $N$. The portrait $B$ is associated with $G$. Consider two randomly chosen nodes at distance $l$. The probability distribution 
$P_{B}(k \mid l)$ is defined as follows:

$$
P_{B}(k \mid l)=\frac{1}{N} B_{l, k}=\frac{k B_{l, k}}{\Sigma_{c} n_{c}^{2}}
$$

where $n_{c}$ is the number of nodes inside each connected component $c$. Accordingly, let $G^{\prime}$ be a network with a total number of nodes $N^{\prime}$ with a portrait $B^{\prime}$, the probability distribution $P_{B^{\prime}}(k \mid l)$ is defined as follows:

$$
P_{B^{\prime}}(k \mid l)=\frac{1}{N^{\prime}} B_{l, k}^{\prime}=\frac{k B_{l k}^{\prime}}{\Sigma_{c} n_{c}^{\prime 2}}
$$

where $n_{c}^{\prime}$ is the number of nodes inside each connected component $c$.

The Network Portrait Divergence between $G$ and $G^{\prime}$ is defined as follows:

$$
D_{J S}\left(G, G^{\prime}\right)=\frac{1}{2}\left(K L\left(P_{B} \| P_{*}\right)+K L\left(P_{B^{\prime}} \| P_{*}\right)\right)
$$

where $P_{*}$ is a combination of $P_{B}$ and $P_{B^{\prime}}$, such as: $P_{*}=\frac{\left(P_{B}+P_{B^{\prime}}\right)}{2}$, and $K L(. \|$.$) is the Kullback-Liebler divergence. The K L$ divergence within two probability distributions $P$ and $P^{\prime}$ is defined as:

$$
\left.K L\left(P(k \mid l)|| P^{\prime}(k \mid l)\right)\right)=\Sigma_{l=0}^{\max \left(d, d^{\prime}\right)} \sum_{k=0}^{N} P \log \left(\frac{P(k \mid l)}{P^{\prime}(k \mid l)}\right)
$$

\section{Experimental evaluation}

Experiments are performed using the movie scripts of six episodes of the Star Wars sage ${ }^{5}$. It is divided into the sequel (original) and prequel trilogies. The sequel trilogy is the first created by George Lucas. It is composed of Episode IV (SW4): A New Hope (1977), Episode V (SW5): The Empire Strikes Back (1980) and Episode VI (SW6): Return of the Jedi (1983). It is followed by the prequel trilogy composed of Episode I (SW1): The Phantom Menace (1999), Episode II

\footnotetext{
${ }^{5}$ Here is a quick summary of the plot: The saga follows Anakin Skywalker, a young child freed from slavery to become a Jedi and endeavored to save the galaxy. Anakin instructed by the Jedi Masters of the light side, married the senator Padme. Unfortunately, the Sith (Palpatine) submits him to the dark side, rebelling and losing against his Master (Obi-Wan). Anakin is saved by the Sith, now ruling over the galaxy, and transformed to Darth Vader. Padme died while giving birth to twins Luke and Leia. Luke becomes a farmer while Leia becomes a princess. Nineteen years later, Obi-Wan met Luke and taught him the Jedi way, while receiving a distress call from the princess Leia, leading the resistance against Palpatine. Joining smuggler Han Solo in the Millenium Falcon they went to save her, and support the resistance. Luke completes his Jedi training, while Solo gets captured by the Sith, who crushes most of the resistance. Vader tries to turn Luke to the dark side when discovering that Luke is his son. Unsuccessful, Palpatine tries to kill Luke, awaking in Vader his old self. Vader turns back against Palpatine and rescues the galaxy.
} 
(SW2): Attack of the Clones (2002), and Episode III (SW3): Revenge of the Sith (2005). The experiment process proceeds as follows. First, the multilayer network of each episode of the saga are extracted. Their basic topological properties are summarised in Table 1. Then, the corresponding portrait for each layer (character, location, and keyword) of each multilayer network are computed individually and discussed. Finally, the distance between each pair of same-type layers between all movies are compared using their portrait divergence.

\begin{tabular}{|c|c|c|c|c|c|c|c|c|c|c|c|c|}
\hline \multirow[b]{2}{*}{ Episode } & \multicolumn{3}{|c|}{ Character Layers } & \multicolumn{4}{|c|}{ Keyword Layers } & \multicolumn{5}{|c|}{ Location Layers } \\
\hline & $N \quad E$ & $D$ & $A$ & $N \quad E$ & $D$ & $T$ & $A$ & $N$ & $E$ & & $T$ & $A$ \\
\hline$\overline{\text { SW1 }}$ & 611090 & 3 & $0.53-0.12$ & 2294492 & 4 & 0.66 & -0.01 & 116 & 26 & 2 & $\overline{0}$ & -0.03 \\
\hline SW2 & 52506 & 3 & $0.43-0.14$ & 2446009 & 4 & 0.52 & -0.01 & 118 & 24 & 2 & 0 & 0.24 \\
\hline SW3 & $56 \quad 476$ & 4 & $0.53-0.008$ & 2334532 & 5 & 0.45 & 0.003 & 152 & 16 & 2 & 0 & -0.19 \\
\hline SW4 & $58 \quad 576$ & 4 & $0.51-0.11$ & 2342201 & 7 & 0.61 & 0.11 & 133 & 428 & 6 & 0.42 & 0.17 \\
\hline SW5 & $45 \quad 504$ & 5 & 0.480 .15 & 211568 & 6 & 0.31 & 0.08 & 145 & 132 & 4 & 0.12 & -0.2 \\
\hline SW6 & 44266 & 4 & 0.420 .34 & 2132648 & 4 & 0.45 & -0.03 & 84 & 30 & 4 & 0.2 & -0.27 \\
\hline
\end{tabular}

Table 1: Global properties of the character, keyword and location layers for each movie, with: number of nodes $(N)$, number of edges $(E)$, diameter $(D)$, transitivity $(T)$, and assortativity $(A)$.

\subsection{Comparing portraits}

Comparing all three layers: A quick look at all three layers: characters in Fig. 2, keywords in Fig. 3, and locations in Fig. 4 clearly shows that each type of layers display a distinct pattern making it recognizable. Character layers display a $k$ between 15 and 30, for a maximum path length between 3 and 5 . Keyword layers do not display much larger path length (up to 6) but the number of nodes are much larger. Despite this larger number of nodes, most nodes are distributed in the lower path corner with degree 1 and 3, maybe suggesting a lot of small components. The character and keyword layers both display clear characteristics common to small-world and scale-free networks $[3$ in their shape such as the elongated form and knot near the center of the portrait. It may be less obvious for the character layer, but one should remember that character graphs are rather small. Location layers diverge the most from small world patterns and seem to greatly vary upon trilogies, while being minimalist in the prequel series. This suggests that locations networks are probably more linear loops in the prequel while they show more complexity in the original trilogy. 


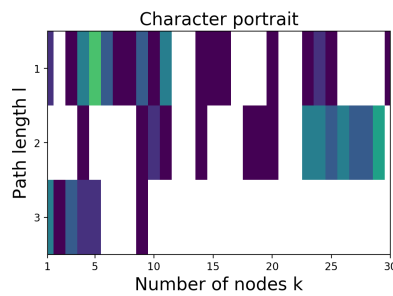

(a)

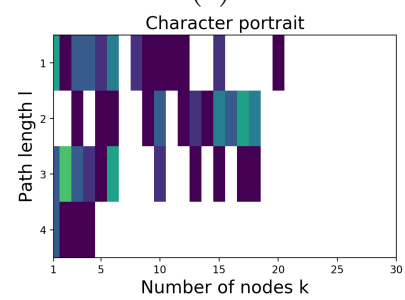

(d)

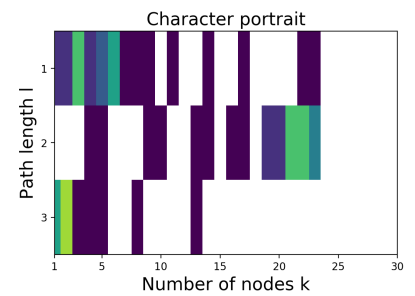

(b)

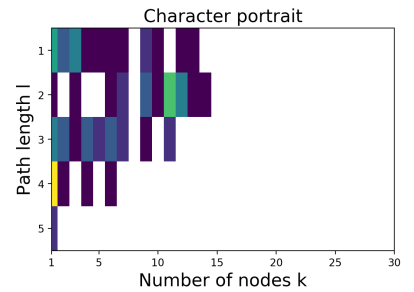

(e)

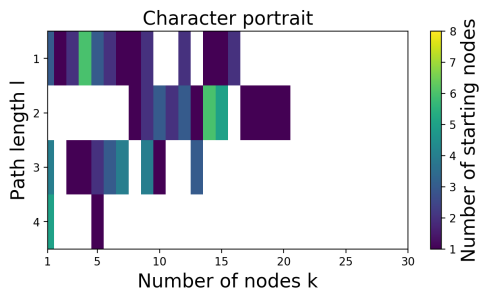

(c)

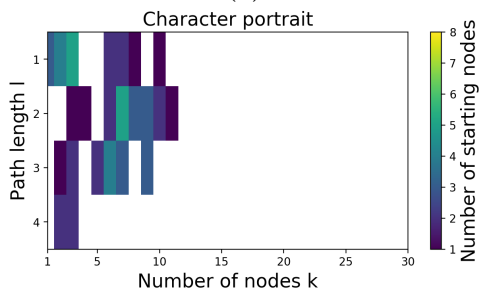

(f)

Fig. 2: Portraits of the characters layer for each movie of the Star Wars saga. (a) SW1, (b) SW2, (c) SW3, (d) SW4, (e) SW5, (f) SW6. The horizontal axis is the node degree $k$. The vertical axis is the distance $l$. Colors are the entries of the portrait matrix $B_{l k}$. The white color indicates $B_{l k}=0$.

Comparing character layers: Each character layer portrait is illustrated in Fig. 2. The overall six portraits display a very similar pattern. SW1 (Fig.22(a)) and SW2 (Fig. 2(b)) - a surprising similarity given that this layer shows a double number of edges as shown in Table 1 - together with SW5 (Fig. 2(e)) and SW6 (Fig. 2(f)) make two similar pairs, with SW3 (Figs. 2(c)) and SW4 (Figs. 2(d)) being somewhat intermediary between them. In SW5 and SW6, a lot of action separates the main characters into different groups with parallel actions, while the first series is rather focused on the main character, Anakin. The aspect ratio of the character layer portraits seems to vary upon the different movies. This has been linked to the small world characteristics of the graph that each portrait summarizes [3]. We can notice a progression from SW1 to SW6 suggesting a "smaller" world effect for episodes 5 and 6 . 


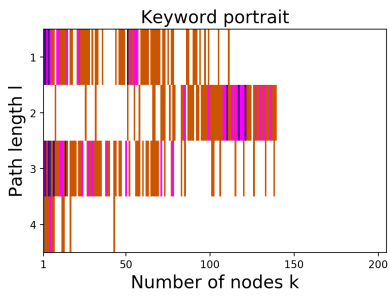

(a)

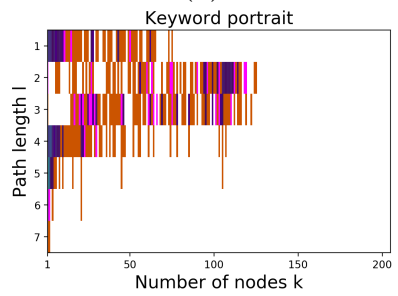

(d)

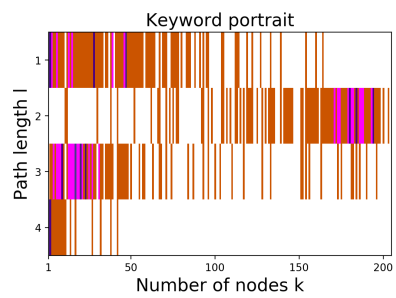

(b)

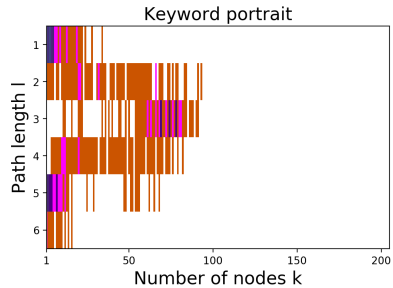

(e)

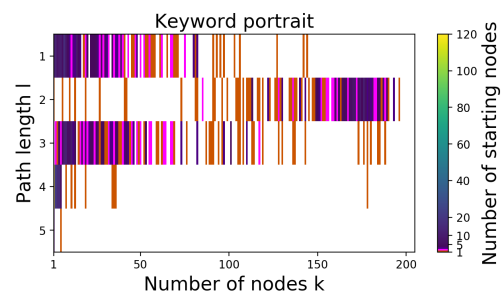

(c)

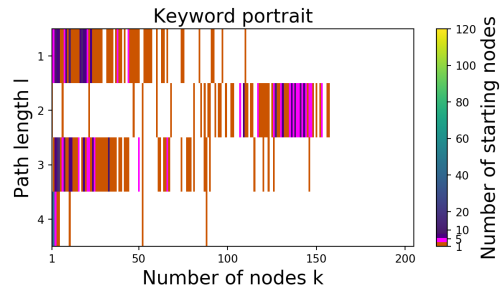

(f)

Fig. 3: Portraits of the keyword layers for each movie of the Star Wars saga. (a) SW1, (b) SW2, (c) SW3, (d) SW4, (e) SW5, (f) SW6. The horizontal axis is the node degree $k$. The vertical axis is the distance $l$. Colors are the entries of the portrait matrix $B_{l k}$. The white color indicates $B_{l k}=0$.

Comparing keyword layers: Each keyword layer portrait is reported in Fig. 3. These layers can be well distinguished from the character layers portrait. Nevertheless, they also exhibit similar patterns across episodes. The aspect ratio of the keyword layer portraits also seems to vary upon the different movies, but not as regularly as the character layer. Especially, the first (Fig. 3 (a)) and last (Fig. 3(f)) episodes seem to share a very similar pattern. SW2 (Fig. 3 (b)) and SW3 (Fig. 3 (c)) also display a close pattern with an elongated aspect ratio, while SW4 (Fig. 3 (d)) and SW5 (Fig. 3(e)) portraits are much shorter. Interestingly, the portrait of this latter one differs in its lowest path length, but all portraits present the "knot" characteristic to scale-free networks. This visual proximity between keyword layers can be expected due to the tendency of language-based graphs to follow Zipf's law [6] [9]. 


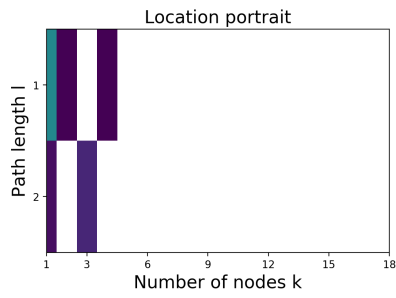

(a)

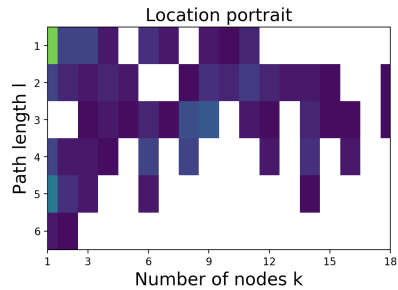

(d)

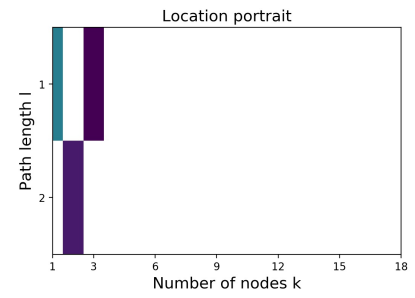

(b)

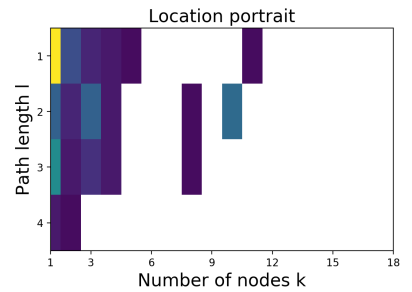

(e)

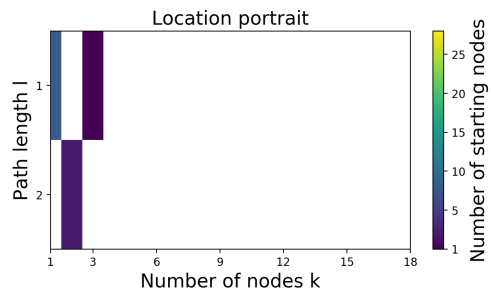

(c)

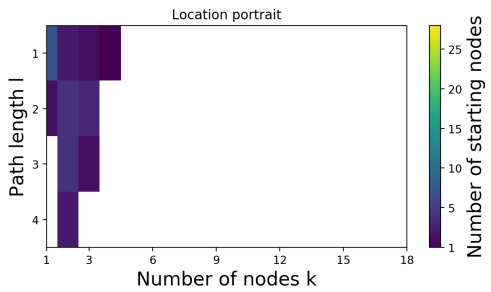

(f)

Fig. 4: Portraits of the locations layer for each movie of the Star Wars saga. (a) SW1, (b) SW2, (c) SW3, (d) SW4, (e) SW5, (f) SW6. The horizontal axis is the node degree $k$. The vertical axis is the distance $l$. Colors are the entries of the portrait matrix $B_{l k}$. The white color indicates $B_{l k}=0$.

Comparing location layers: Each location layer portrait is illustrated in Fig. 4. At a glance, one can recognize a pattern very different to the character and keyword layers. The pattern is common to all three episodes of the prequel trilogy (Fig. $4(\mathrm{a}-\mathrm{c})$ ). It shows very low maximum path length and degree. This strongly suggest the presence of chain-type topology. As a consequence, the cut of these three movies between locations may be quite linear. Indeed this trilogy mostly follows the actions of Anakin and Obi-wan, very often together. The three episodes of the original trilogy display a more complex structure. The structure is more complex in the first (Fig. 4(d)) of these episodes, then gradually simplify to the last episode (Fig. 4(f)). In SW4, iconic star ship locations are introduced, such as the Millenium Falcon and the Death Star, and the movie cuts a lot scenes between star ships and other locations (this can be confirmed from the number of edges in Table 1). The rhythm is also different in the original trilogy, which often separates its main characters such that actions happen in parallel. Frequent cuts between them generate transitions that are less linear. This trend seems to be less adopted after the fourth episode.

\subsection{Comparing portrait divergence}

Fig. 5 illustrates the network portraits divergence between each SW movie. Fig. 5 (a) shows the divergence between character layers, Fig. 5(b) shows divergence between keyword layers, and Fig. 5(c) shows the divergence between location layers. 


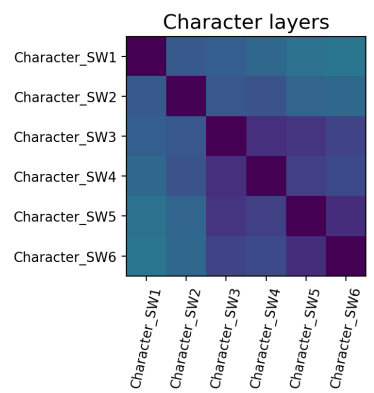

(a)

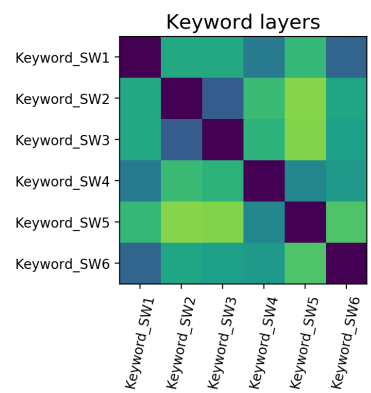

(b)

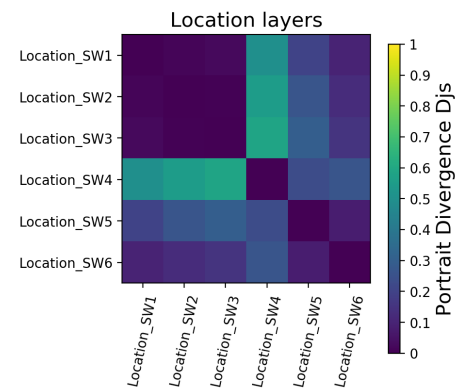

(c)

Fig. 5: Portrait divergence of the characters, keywords, and locations networks in the 6 episodes of the Star Wars saga. (a) Character layers. (b) Keyword layers. (c) Location layers. Each cell of the heat-map represents the portrait divergence between a couple of episodes.

Overall comparison: Comparing overall layer divergence suggests that relationships in character layers are very similar along the saga. This confirms the intuition we can have at a glance from the portraits figures. Location layers also show an overall high similarity. The keyword heat-map departs from the others. Indeed, differences are more marked between the keyword layers that exhibit a more patchy structure. This is surprising considering that the visualization of each portrait made one movie to another looks very similar. It is however harder to make sense of why the fifth episode stands out. Finally, the separation between each trilogy appears clearly in the location layer. Note that the pairwise character layer similarity also makes sense considering the movies.

Divergence between characters layers: From Fig. 5(a), the prequel trilogy (SW1-3) shows a low divergence between characters. Relationships between characters of SW1 appear more closely related to SW2 $\left(D_{J S}=0.279\right)$ than to SW3 $\left(D_{J S}=0.299\right)$. That may be due the appearance and disappearance of some key characters. For instance, Shmi dies in SW2. In SW3, Anakin changes side to become Vader, and the clones start to appear. This last episode shows an even lower divergence with the sequel: the clones being instrumental in the original series may help shaping a common structure of relationships. This may also confirm the efforts taken in the third episode of the prequel to link characters with the original series.

In the orginal trilogy, SW5 and SW6 show the lowest divergence $\left(D_{J S}=0.132\right)$. Indeed, individual inspection shows that a lot of the links are shared in both episodes. SW4 appears the most diverging with the other movies in the trilogy. This make sense since the movie was made so that it could have been a stand alone movie at first. It shows its closest relationship with SW3 $\left(D_{J S}=0.134\right)$, once more underlining the special care taken to connect the prequel to the sequel from the character relationships point of view. SW1 and SW6 show the highest 
divergence. This is not surprising since at this point the story of the prequel and original trilogies are completely different in their characters and plots.

Divergence between keyword layers: The keyword layers show the highest divergence among the different movies (Fig. 5 (b)). In both trilogies, the divergence appears lower for a pair of movies, SW2 and SW3 in the prequel $\left(D_{J S}=0.29\right)$, and SW4 and SW5 in the sequel. SW1 shows its lower divergence with SW4 $\left(D_{J S}=0.4\right)$ and SW6 $\left(D_{J S}=0.33\right)$. It remains difficult to conclude what in the structure of the movies can lead to a similar relationship structure in keywords between those three. We suspect the frequent association of the key terms young and master in these movies to play some important role.

Divergence between location layers: Observing Fig. 5(c), the portrait divergence shows very high similarity within the structure of locations of the first prequel. The original series also shows a low divergence between movies. We observe a noticeable difference between the prequel trilogy and SW4, specifically, between SW3 and SW4 with $D_{J S}=0.585$. From SW4, a lot of scenes start taking place in the Millenium Falcon, and death stars, giving a specific rhythm in cuts and locations. Nonetheless, divergence is rather low between the prequel and the last two episodes of the series. Remembering that all information is extracted from the scripts, we may suspect here different influences the movie director may have. Indeed, SW4 was written to possibly be a standalone movie, while the following SW5 and SW6 scripts were written in a short period of span, planned to complete the trilogy. This concerns even more the prequel trilogy which was planned from the beginning to be a unified trilogy.

\section{Discussion and conclusion}

In this work, a multilayer network model is used to represent the main elements of a movie script: characters, key elements of the conversations (keywords), and locations of the scenes. Investigations based on the single-layer components of the model are performed to relate the similarity of the 6 Star Wars Saga episodes to the distance between the layers based on portrait divergence.

Preliminary results using portrait and portrait divergence measures are quite encouraging. Indeed, the analysis of the movie networks confirms a good correspondence between characters of the prequel and sequel trilogies; and a difference between locations in the prequel and sequel trilogy. Each of the prequel and sequel trilogies shows a good relationship between characters; and high similarity between locations. Results show similarity between topic relationships (keywords) in the movies SW2 and SW3; and also of SW1 with SW4 and SW6. Otherwise, other episodes appear to be dissimilar, mainly, the relation connecting keywords of SW5 with SW2 and SW3. Although more experiments are needed to fully assess that movie similarities can be discovered by measuring their network distances, this work opens multiple research directions.

Note that the current results are only based on the sole script of each movie. The script of SW4 was written in the late 70's, the two following movies in the 80 's and the prequel in the late 90 's/2000's. How movies are scripted have 
definitely changed over this span, and may impact our results. To even go further, we can enrich the model. Previous works have also formulated multimedia cues for movie analysis [16] including its visual features often important for recommendation systems [27]. We may further enrich the model, using for example sentiment analysis. To further evaluate how the network similarity performs, we wish to further proceed with user evaluation, and script-based topical [5] and style [10] similarity.

Our processing scheme is currently exploiting layers of the multilayer network as separated single-layer networks. One interesting task would be to compare with distance measures of multilayer networks. Since the Kullback-Liebler divergence is not a metric and does not respect the triangular inequality, although tempting, we have found irrelevant to average divergence over all layers and measure overall similarity between movies. Alternative strategies could be to project the whole multilayer network into a single-layer knowledge graph, but such projection remains unsatisfying. We further need to investigate pure multilayer approaches and include in the comparison the effect of transition layers. We can try embedding-based comparisons [1], and investigate proper multilayer metrics such as entanglement 22] or nodes and edge coupling [7] between movies. This however requires to align named entities between layers and movies, such that they refer to the same entity. This is not straightforward since we have at least two entities with an ambiguous definition which are Anakin/Vader and Amidala/Padme. Finally, we believe this methodology to be useful once incorporated into recommendation systems to increase their efficiency [19] since we provide a different definition of a genre based on actual elements of the movie content [8].

\section{References}

1. Bagavathi, A., Krishnan, S.: Multi-net: Scalable multilayer network embeddings. arXiv preprint arXiv:1805.10172 (2018)

2. Bagrow, J.P., Bollt, E.M.: An information-theoretic, all-scales approach to comparing networks. Applied Network Science 4(1), 45 (2019)

3. Bagrow, J.P., Bollt, E.M., Skufca, J.D., Ben-Avraham, D.: Portraits of complex networks. EPL (Europhysics Letters) 81(6), 68004 (2008)

4. Blei, D.M., Ng, A.Y., Jordan, M.I.: Latent dirichlet allocation. Journal of machine Learning research 3(Jan), 993-1022 (2003)

5. Bougiatiotis, K., Giannakopoulos, T.: Content representation and similarity of movies based on topic extraction from subtitles. In: Proceedings of the 9th Hellenic Conference on Artificial Intelligence. pp. 1-7 (2016)

6. Cancho, R.F.I., Solé, R.V.: The small world of human language. Proceedings of the Royal Society of London. Series B: Biological Sciences 268(1482), 2261-2265 (2001)

7. Cozzo, E., Kivelä, M., De Domenico, M., Solé-Ribalta, A., Arenas, A., Gómez, S., Porter, M.A., Moreno, Y.: Structure of triadic relations in multiplex networks. New Journal of Physics 17(7), 073029 (2015)

8. Deldjoo, Y., Schedl, M., Elahi, M.: Movie genome recommender: A novel recommender system based on multimedia content. In: 2019 International Conference on Content-Based Multimedia Indexing (CBMI). pp. 1-4. IEEE (2019) 
9. Grabska-Gradzińska, I., Kulig, A., Kwapień, J., Drożdż, S.: Complex network analysis of literary and scientific texts. International Journal of Modern Physics C 23(07), 1250051 (2012)

10. Kovacs, B., Kleinbaum, A.M.: Language-style similarity and social networks. Psychological Science 31(2), 202-213 (2020)

11. Labatut, V., Bost, X.: Extraction and analysis of fictional character networks: A survey. ACM Computing Surveys (CSUR) 52(5), 1-40 (2019)

12. Lee, O.J., Jo, N., Jung, J.J.: Measuring character-based story similarity by analyzing movie scripts. In: Text2Story@ ECIR. pp. 41-45 (2018)

13. Lee, O.J., Jung, J.J.: Explainable movie recommendation systems by using storybased similarity. In: IUI Workshops (2018)

14. Markovič, R., Gosak, M., Perc, M., Marhl, M., Grubelnik, V.: Applying network theory to fables: complexity in slovene belles-lettres for different age groups. Journal of Complex Networks 7(1), 114-127 (2018)

15. Mourchid, Y., Renoust, B., Cherifi, H., El Hassouni, M.: Multilayer network model of movie script. pp. 782-796. Springer (2018)

16. Mourchid, Y., Renoust, B., Roupin, O., Văn, L., Cherifi, H., El Hassouni, M.: Movienet: a movie multilayer network model using visual and textual semantic cues. Applied Network Science 4(1), 1-37 (2019)

17. Nadeau, D., Sekine, S.: A survey of named entity recognition and classification. Lingvisticae Investigationes 30(1), 3-26 (2007)

18. Park, S.B., Oh, K.J., Jo, G.S.: Social network analysis in a movie using characternet. Multimedia Tools and Applications 59(2), 601-627 (2012)

19. Reddy, S., Nalluri, S., Kunisetti, S., Ashok, S., Venkatesh, B.: Content-based movie recommendation system using genre correlation. In: Smart Intelligent Computing and Applications, pp. 391-397. Springer (2019)

20. Renoust, B., Kobayashi, T., Ngo, T.D., Le, D.D., Satoh, S.: When face-tracking meets social networks: a story of politics in news videos. Applied Network Science 1(1), 4 (2016)

21. Renoust, B., Le, D.D., Satoh, S.: Visual analytics of political networks from facetracking of news video. IEEE Transactions on Multimedia 18(11), 2184-2195 (2016)

22. Škrlj, B., Renoust, B.: Patterns of multiplex layer entanglement across real and synthetic networks. In: International Conference on Complex Networks and Their Applications. pp. 671-683. Springer (2019)

23. Stiller, J., Nettle, D., Dunbar, R.I.: The small world of shakespeare's plays. Human Nature 14(4), 397-408 (2003)

24. Tan, M.S., Ujum, E.A., Ratnavelu, K.: A character network study of two sci-fi tv series. vol. 1588, pp. 246-251. AIP (2014)

25. Tantardini, M., Ieva, F., Tajoli, L., Piccardi, C.: Comparing methods for comparing networks. Scientific Reports 9(1), 17557 (2019), https://doi.org/10. 1038/s41598-019-53708-y

26. Waumans, M.C., Nicodème, T., Bersini, H.: Topology analysis of social networks extracted from literature. PloS one 10(6), e0126470 (2015)

27. Zhou, H., Hermans, T., Karandikar, A.V., Rehg, J.M.: Movie genre classification via scene categorization. In: Proceedings of the 18th ACM international conference on Multimedia. pp. 747-750 (2010) 